\title{
An Efficient Explicit Marching on in Time Solver for Magnetic Field Volume Integral Equation
}

\author{
Sadeed Bin Sayed", H. Arda Ulku*, , and Hakan Bagci ${ }^{*}$ \\ *Division of Computer, Electrical, and Mathematical \\ Sciences and Engineering \\ King Abdullah University of Science and Technology \\ (KAUST), Thuwal, Saudi Arabia \\ ${ }^{\dagger}$ Department of Electronics Engineering \\ Gebze Institute of Technology \\ Kocaeli, Turkey \\ haulku@gyte.edu.tr
}

\{sadeed.sayed, huseyin.ulku, hakan.bagci\}@kaust.edu.sa

\begin{abstract}
An efficient explicit marching on in time (MOT) scheme for solving the magnetic field volume integral equation is proposed. The MOT system is cast in the form of an ordinary differential equation and is integrated in time using a $P E(C E)^{m}$ multistep scheme. At each time step, a system with a Gram matrix is solved for the predicted/corrected field expansion coefficients. Depending on the type of spatial testing scheme Gram matrix is sparse or consists of blocks with only diagonal entries regardless of the time step size. Consequently, the resulting MOT scheme is more efficient than its implicit counterparts, which call for inversion of fuller matrix system at lower frequencies. Numerical results, which demonstrate the efficiency, accuracy, and stability of the proposed MOT scheme, are presented.
\end{abstract}

\section{INTRODUCTION}

Transient electromagnetic scattering from inhomogeneous dielectric objects can be analyzed by solving time domain magnetic field volume integral equations (TD-MFVIE). It has been shown in [1] that discretization of the MFVIE results in a well-conditioned system regardless of the dielectric contrast of the scatterer. "Traditional" marching on in time (MOT)-based MFVIE solvers expand the unknown magnetic fields using fully linear curl conforming basis functions in space [2] and polynomial interpolators in time [3] This expansion is inserted into the TD-MFVIE; Galerkin or point testing [4] the resulting equation at discrete times yields a lower triangular matrix system. This system is solved for unknown expansion coefficients in a recursive manner via forward substitution in time. The resulting MOT scheme is implicit and calls for solution of a (smaller) matrix system at every time step. This matrix system becomes fuller as time step size gets larger under low frequency excitations.

To alleviate this bottleneck, in this work, an explicit MOT scheme for solving the TD-MFVIE is described. First, the TDMFVIE is cast in the form of an ordinary differential equation. The unknown magnetic field is expanded in space using fully linear curl conforming basis functions [2]. The expansion is inserted into the TD-MFVIE and the resulting equation is tested in space. The final semi-discrete equation is integrated in time using a $P E(C E)^{m}$ multistep scheme. At the prediction/correction stages of every time step, a system with
Gram matrix is solved [5]. For point testing in space, the Gram matrix consist of four diagonal sub-matrix blocks; its inverse has the same structure and is computed and stored before time marching. For Galerkin testing in space, Gram matrix is unstructured but still sparse. In both cases, the matrix inversion is very efficient regardless of the time step size. In both testing cases, the resulting MOT scheme uses time steps as large as those of corresponding "traditional" implicit MOT schemes without sacrificing from stability and accuracy. Indeed, numerical results demonstrate that the proposed explicit MOT solver is more efficient in solving low frequency problems, and still maintains the stability and accuracy of the solution.

\section{FORMULATION}

Let $V$ denote the volume of an inhomogeneous dielectric scatterer residing in an unbounded background medium, both having a permeability of $\mu_{0}$. Permittivities of the scatterer and background medium are $\varepsilon(\mathbf{r})$ and $\varepsilon_{0}$, respectively. Incident field $\mathbf{H}^{\text {inc }}(\mathbf{r}, t)$, which is band-limited to $f_{\max }$ and vanishingly small $\forall \mathbf{r} \in V$ and $t \leq 0$, excites the scatterer. Upon excitation, magnetic field $\mathbf{H}(\mathbf{r}, t)$ is induced in $V$. Fields $\mathbf{H}(\mathbf{r}, t)$ and $\mathbf{H}^{\text {inc }}(\mathbf{r}, t)$ satisfy the time differentiated TD-MFVIE:

$$
\begin{aligned}
& 4 \pi \partial_{t} \mathbf{H}^{\mathrm{inc}}(\mathbf{r}, t)=4 \pi \partial_{t} \mathbf{H}(\mathbf{r}, t) \\
& +\int_{V} \hat{\mathbf{R}} \times \kappa\left(\mathbf{r}^{\prime}\right)\left(\frac{\nabla^{\prime} \times \partial_{t^{\prime}}^{2} \mathbf{H}\left(\mathbf{r}^{\prime}, t^{\prime}\right)}{c R}+\frac{\nabla^{\prime} \times \partial_{t^{\prime}} \mathbf{H}\left(\mathbf{r}^{\prime}, t^{\prime}\right)}{R^{2}}\right)_{t^{\prime}=t-R / c} d v^{\prime} .
\end{aligned}
$$

Here, $\kappa(\mathbf{r})=1-\varepsilon_{0} / \varepsilon(\mathbf{r})$ is the dielectric contrast, $R=\left|\mathbf{r}-\mathbf{r}^{\prime}\right|$ is the distance between source and observation points, $\mathbf{r}$ and $\mathbf{r}^{\prime}$, and $c=1 / \sqrt{\varepsilon_{0} \mu_{0}}$ is the speed of light in the background medium. To numerically solve $(1), \mathbf{H}(\mathbf{r}, t)$ is expanded in terms of fully linear curl conforming basis functions $\mathbf{f}_{n}(\mathbf{r})$ [2]:

$$
\mathbf{H}(\mathbf{r}, t)=\sum_{n=1}^{2 N}\{\mathbf{I}(t)\}_{n} \mathbf{f}_{n}(\mathbf{r}) .
$$

Here, $\mathbf{I}(t)$ stores the time dependent unknown expansion coefficients. Expansion (2) is inserted in (1) and the resulting equation is tested using $\mathbf{t}_{m}(\mathbf{r}), m=1: 2 N$, at times $t=j \Delta t$, where $\Delta t$ is the time step. To facilitate the computation of retarded time integrals in tested version of (1), temporal interpolator $T(t-i \Delta t)$ [3] is used between the samples of $\mathbf{I}(t)$. 
This discretization scheme "converts" (1) into the matrix system:

$$
\mathbf{G} \tilde{\mathbf{I}}_{j}=\mathbf{Z}_{0} \mathbf{I}_{j}+\mathbf{V}_{j}^{\mathrm{inc}}+\sum_{i=0}^{j-1} \mathbf{Z}_{j-i} \mathbf{I}_{i}
$$

where $\quad\left\{\mathbf{I}_{i}\right\}_{n}=I_{n, i}$ and $\left\{\tilde{\mathbf{I}}_{i}\right\}_{n}=\left.\partial_{t} I_{n}(t)\right|_{t=i \Delta t}$ are unknown expansion coefficients and their derivatives. The entries of the other vectors and matrices in (3) are given by:

$$
\begin{aligned}
& \left\{\mathbf{V}_{j}^{\mathrm{inc}}\right\}_{m}=\left\{\mathbf{V}^{\mathrm{inc}}(j \Delta t)\right\}_{m} \\
& \{\mathbf{G}\}_{m, n}=\int_{V} \mathbf{t}_{m}(\mathbf{r}) \cdot \mathbf{f}_{n}(\mathbf{r}) d v \\
& \left\{\mathbf{Z}_{j-i}\right\}_{m, n}=\left\langle\mathbf{t}_{m}(\mathbf{r}), \nabla^{\prime} \times \mathbf{f}_{n}\left(\mathbf{r}^{\prime}\right), T(t-i \Delta t)\right\rangle_{t=j \Delta t}
\end{aligned}
$$

Here, the operation $\langle\cdot, \cdot, \cdot\rangle$ is defined as

$$
\begin{aligned}
& \left\langle\mathbf{x}(\mathbf{r}), \mathbf{y}\left(\mathbf{r}^{\prime}\right), z(t)\right\rangle_{t=j \Delta t}=\frac{1}{4 \pi} \int_{V} \mathbf{x}(\mathbf{r}) \cdot \int_{V} \kappa\left(\mathbf{r}^{\prime}\right) \mathbf{y}\left(\mathbf{r}^{\prime}\right) \\
& \times\left.\hat{\mathbf{R}}\left(\frac{\partial_{t}^{2} z(t-R / c)}{c R}+\frac{\partial_{t} z(t-R / c)}{R^{2}}\right) d v^{\prime} d v\right|_{t=j \Delta t} .
\end{aligned}
$$

The matrix system (3) is in the form an ordinary differential equation and is integrated in time using a $P E(C E)^{m}$ multistep method [5] to compute the samples of the unknown coefficients $\left\{\mathbf{I}_{i}\right\}_{n}$. At the prediction/correction stages of every time step, matrix equation (3) is solved. For point testing, $\mathbf{G}^{-1}$ consists of four diagonal sub-matrix blocks; and is computed very efficiently and stored before time marching. For Galerkin testing, i.e., $\mathbf{t}_{m}(\mathbf{r})=\mathbf{f}_{m}(\mathbf{r})$, Gram matrix is unstructured but still sparse. In both cases, the matrix system (3) can be inverted very efficiently regardless of the time step size.

\section{NUMERICAL RESULTS}

In this section, the efficiency, accuracy, and stability of the proposed MOT scheme are demonstrated using numerical examples. In all examples, the excitation is a plane wave with magnetic field

$$
\mathbf{H}^{\mathrm{inc}}(\mathbf{r}, t)=\hat{\mathbf{y}} G(t-\mathbf{r} \cdot \hat{\mathbf{z}} / c) .
$$

Here, $\quad G(t)=\cos \left(2 \pi f_{0}\left[t-t_{0}\right]\right) \exp \left(-\left[t-t_{0}\right]^{2} / 2 \sigma^{2}\right), \quad$ is a modulated Gaussian pulse with center frequency $f_{0}$, effective bandwidth $f_{\mathrm{bw}}$, duration $\sigma=3 /\left(2 \pi f_{\mathrm{bw}}\right)$, and delay $t_{0}=10 \sigma$. The $P E(C E)^{m}$ scheme uses the fourth order Adam-Bashworth and backward difference formulas at the predictor and corrector stages, respectively.

First, the efficiency of the proposed solver in solving low frequency problems is demonstrated. The scatterer is a unit dielectric sphere with relative permittivity $\varepsilon_{r}=3$. It is centered at the origin. The parameters of the incident field are $f_{0}=8$ $\mathrm{MHz}$ and $f_{\mathrm{bw}}=4 \mathrm{MHz}$. The order of $T(t)$ is two and $\Delta t=4$ ns. Point testing is used in space. Fig. 1(a) compares the CPU times required for the proposed solver and its implicit counterpart, which uses the same parameters, for different values of $N$. Clearly, the explicit solver is faster. Fig. 1(b) presents the radar cross section (RCS), which is obtained by Fourier transforming the time domain results of the proposed solver at $8 \mathrm{MHz}$ for $\phi=0^{\circ}$ and $\theta=\left[0^{\circ}, 180^{\circ}\right]$ for $N=4448$. Results agree well with Mie series solution.
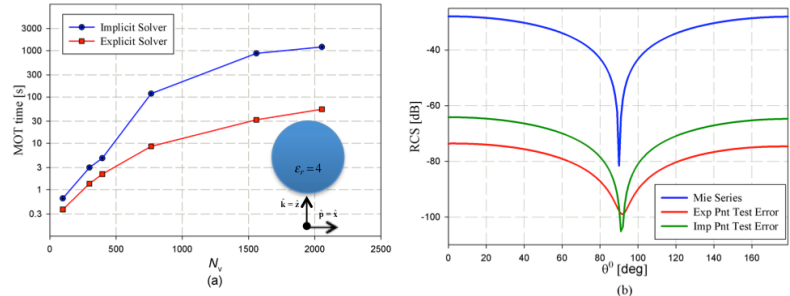

Fig. 1. (a) CPU time for marching, (b) RCS.

Next, the accuracy and stability of the proposed solver for scatterers with high contrast values are demonstrated. The scatter is a unit sphere with $\varepsilon_{r}=50$. The parameters of the incident field are $f_{0}=5 \mathrm{MHz}$ and $f_{\mathrm{bw}}=2.5 \mathrm{MHz}$. The unknown field is discretized using 4448 basis function. The order of $T(t)$ is two and $\Delta t=5 \mathrm{~ns}$. Galerkin testing is used in space. Fig. 2(a) shows that the solution is stable and Fig 2(b) shows that the error in RCS at $5 \mathrm{MHz}$ is less than $40 \mathrm{~dB}$.
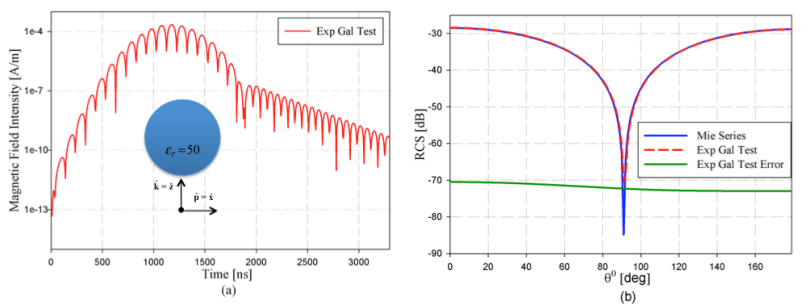

Fig. 2.(a) Solution at $(0.51,-0.64,0.12) \mathrm{m}$, (b) RCS

\section{REFERENCES}

[1] J. Markkanen, C. C. Lu, X. Cao and P. Ylä-Oijala, "Analysis of volume integral equation formulations for scattering by high-contrast penetrable objects, "IEEE Trans. Antennas Propag., vol. 60, no.5, pp. 2367-2374, May 2012.

[2] J. P. Webb, "Hierarchal vector basis functions of arbitrary order for triangularand tetrahedral finite elements," IEEE Trans. Antennas Propagat., vol. 47, no. 8, pp. 1244-1253, Aug. 1999.

[3] G. Manara, A. Monorchio, and R. Reggiannini, "A space-time discretization criterion for a stable time-marching solution of the electric field integral equation," IEEE Trans. Antennas Propag., vol. 45, pp. 527-532, Mar. 1997.

[4] A. F. Peterson, "Performance of stable volume MFIE formulations for high contrast dielectric targets," Antennas and Propagation Society International Symposium (APSURSI), IEEE, 2014.

[5] H. A. Ulku, H. Bagci, and E. Michielssen, "Marching on-in-time solution of the time domain magnetic field integral equation using a predictor-corrector scheme," IEEE Trans. Antennas Propag., vol. 61, no. 8, pp. 4120-4131, Aug. 2013. 